\title{
Surgically-assisted Rapid Maxillary Expansion under Local Anesthesia
}

\author{
Cansu Gul Koca1 and Ihsan Sami Guvenc ${ }^{2}$ \\ Department of Oral and Maxillofacial Surgery1 / Orthodontics², Faculty of Dentistry, Usak University, Usak, Turkey
}

\begin{abstract}
Developmental, traumatic and congenital factors, among many others, may lead to transverse maxillary deficiency (TMD). TMD can be corrected by orthodontic treatment, and may also require surgical intervention. The surgical technique is used in maxillary hypoplasia seen in cleft palate, crowding in maxillary teeth, which in cases where maxilla needs to be expanded more than $5 \mathrm{~mm}$. Although it is a frequently used technique, there is no consensus on the operative technique and the apparatus used during the operation. Whether or not to separate, the pterygomaxillary junction is also one of the most common subjects of debate in this regard.

In this case presentation, the transverse expansion of the maxilla was completed by means of surgically-assisted rapid maxillary expansion operation performed under local anesthesia without separating the pterygomaxillary junction and nasal septal osteotomy; and the patient, in whom orthodontic treatment had been completed, made a successful recovery without complications.
\end{abstract}

Key Words: Maxillary expansion, Osteotomy, Pterigopalatal junction.

How to cite this article: Gul C, Sami I. Surgically-assisted rapid maxillary expansion under local anesthesia. J Coll Physicians Surg Pak 2019; 29 (Supplement 2):S129-S131.

\section{INTRODUCTION}

Developmental, traumatic and congenital factors, among many others, can cause transverse maxillary deficiency. ${ }^{1}$ In addition to aesthetic concerns, maxillary deficiency may also lead to apnea, difficulty in chewing, dental crowding and nasal obstruction. ${ }^{1}$ Surgically-assisted rapid maxillary expansion (SARME) is performed to expand the maxilla. ${ }^{2}$ Although SARME is a frequently used technique, whether or not to separate the pterygomaxillary junction is also one of the most common subjects of debate in this regard. ${ }^{1}$

In the present case, the transverse expansion of maxilla was completed by means of SARME operation performed under local anesthesia without separating the pterygomaxillary junction; and the patient, in whom orthodontic treatment had been completed, made a successful recovery without complications.

\section{CASE REPORT}

The lateral cephalometric radiograph, model analysis and clinical examination of a female patient at 17 years and 1 month of age, who presented to the Department of Orthodontics with a complaint of crowding in the maxillary anterior area, revealed transverse maxillary deficiency with an indication for SARME.

Correspondence to: Cansu Gul Koca, Faculty of Dentistry,

Oral and Maxillofacial Surgery, Usak University, Usak, Turkey

E-mail: cansu.koca@usak.edu.tr

Received: February 06, 2019; Revised: July 01,2019;

Accepted: July 10, 2019
Intraoral examination of the patient, on the other hand, revealed posterior crossbite, a narrow and deep maxillary arch, decreased overjet, and skeletal class 1 malocclusion following cephalometric analysis. The upper and lower incisor angles and the perpendicular dimension size were observed to be within normal limits. The model analysis revealed a need for $8.1 \mathrm{~mm}$ space in the mandible and a $2.1 \mathrm{~mm}$ space in the maxilla, and the analysis of the panoramic radiograph revealed that all the third molars were present but impacted in the upper jaw. A preoperative and intraoral view of the maxillary arch and the jaw cast are shown in Figure 1. Plan was made for the SARME correction of the transverse maxillary deficiency.

The incision was made at the deepest part of the buccal sulcus between the bilateral maxillary canine and the first molar, after local anesthesia was achieved. A mucoperiosteal flap was elevated from the piriform aperture to the zygomatic buttress. During this procedure, care was taken not to damage the nasal mucosa. Holes were bored in the bone tissue from piriform aperture to zygomatic buttress posterior with the drill at the apical area approximately $5 \mathrm{~mm}$ from the tooth roots and the holes were joined using a fissure drill. Following this procedure, the osteotomy was continued until the tuber maxilla using gouge and hammer. Anterior osteotomy of the maxilla was initiated after the completion of maxillary lateral osteotomies. Following the vertical incision made parallel to the maxillary anterior frenulum, midline osteotomy was initiated from the middle of the central 
teeth. After the osteotomy in the maxillary anterior region had been completed, the expansion apparatus, which had been fixed to the maxilla one week before the surgery, was activated by spinning 10 quarter-rounds forward and 5 quarter-rounds backward ( $0.25 \mathrm{~mm}$ for each spin). After the bleeding was taken under control, the surgical site was sutured.

In order to prevent postoperative infection, the patient was prescribed antibiotics, analgesics, and mouthwash. After two days of latency period, the appliance was activated two times a day (totally $0.5 \mathrm{~mm}$ ). The expansion process continued until overcorrection was achieved on both sides for maxillary stenosis. After sufficient expansion was achieved, the Hyrax screw was sealed with acrylic resin. The apparatus was kept cemented in the mouth for 5 months during the retention process. After that, the apparatus was removed and used a mobile for one month. After the skeletal correction was completed following the expansion process, the dental correction was started. Fixed orthodontic treatment was started using 0.022 " slot MBT brackets.

Maxillary stenosis problem was resolved. Class 1 molar and the canine relationship was achieved in the patient. Skeletal and dental values at the beginning of the treatment did not show significant change, and the smile line was corrected. The maxillary expansion achieved with the procedure under local anesthesia, which has been depicted in Figure 2.

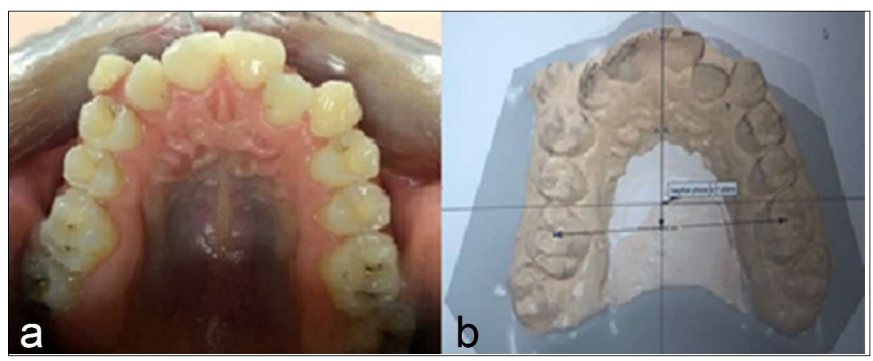

Figure 1: (a) Preoperative intraoral view. (b) The cast of the maxillary arch. These indicate the need for rapid maxillary expansion.

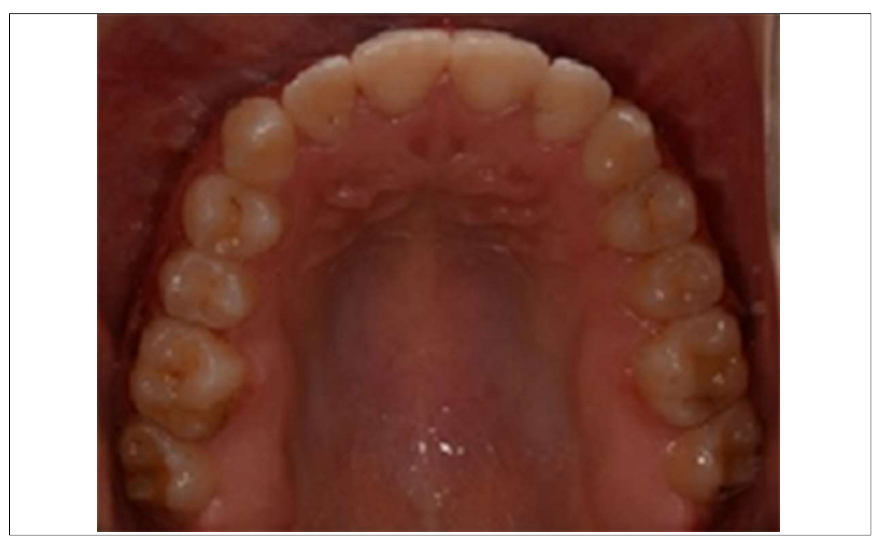

Figure 2: Postoperative intraoral view of the maxillary arch showing the resultant maxillary expansion.

\section{DISCUSSION}

Rapid maxillary expansion is a bone distraction procedure that was first introduced by llizarov's works. It is particularly preferred in cases of TMD, as well as in cases of significant maxillary hypoplasia due to mandibular hyperplasia, crowding of maxillary teeth, buccal corridor that forms during smiling, and in cases where maxilla needs to be expanded more than $5 \mathrm{~mm}$. Conventional rapid maxillary expansion performed on adult patients with completed ossification in midpalatal sutures leads to a number of adverse effects. These effects can be listed as tipping of teeth, absence of skeletal effect, periodontal problems, and pain occurrence. Although SARME is a frequently used method, there is still no consensus on the apparatus used, the amount of relapse, overcorrection, and the surgical technique employed. The main underlying reason for differing views on the surgical technique is that different anatomical regions are considered to be the main resistance areas that prevent the transverse development of the maxilla. ${ }^{3}$ These resistance areas are zygomaticomaxillary suture, pterigomaxillary suture, midpalatal suture, and piriform aperture. On this subject, many researchers performed operations on the resistance areas they proposed, and contributed their findings to the literature. Some researchers considered the median palatal suture as the main point of resistance and only performed osteotomy in this area. ${ }^{3}$ However, this view had become obsolete in time. There are also researchers who performed bilateral osteotomy from aperture to tuber maxilla by adding or not adding midpalatal suture in the osteotomy line, and achieved successful results. 4,5

Pterygomaxillary junction is one of the resistance areas on the maxilla, and due to its close proximity to vital anatomical structures, whether to include it in SARME operations still remains a matter of debate. There are researchers who achieved successful results with or without including it. One such research was carried out by Berger et al., 6 who argued that an advanced surgical technique, in which subtotal LeFort 1 and midpalatal suture osteotomy were performed jointly, prevents relapse in the long-term. ${ }^{6}$ As the surgical technique becomes more advanced, the risk of complications also increases. ${ }^{7}$ Pogrel et al. performed lateral wall osteotomy along with midpalatal suture osteotomy up to the zygomaticomaxillary suture and reported at the end of a one year follow-up that the results were satisfactory. 8 In their study, Zandi et al. compared the operation findings of two groups, one of which included pterygomaxillary junction in the operation while the other did not. Nasal base width, interdental root distance, palatal bone width and interdental cusp distance were measured at the first molar and first premolar areas right before the operation and immediately after the end of the consolidation period. Measurements revealed no statistically significant difference between the groups that included 
and excluded the pterygomaxillary junction in the operation. In other words, in both groups, the maxilla was observed to have homogeneously expanded in the posterior and anterior directions, while maximum expansion was seen in the dental arch. ${ }^{9}$

As we have previously mentioned, separation of the pterygomaxillary junction is one of the most frequently debated topics on the subject of SARME. Separation of the pterygomaxillary junction may lead to complications that may arise due to trauma to the pterygomaxillary artery or cranial nerves, the need for general anesthesia, and prolonged operation times, etc. It is also reported that not separating pterygomaxillary junction, on the other hand, may result in a greater expansion in the maxillary anterior region compared to the posterior region, a weakening in the periodontal support, and a feeling of greater pain during the expansion of the maxilla. 10

In conclusion, it is said that modified SARME technique is a method that can be performed easily under local anesthesia with fewer postoperative complications and ease of application. Although there is no consensus regarding the employed surgical technique, type of apparatus, expansion protocol, and different protocols can be determined by the clinician for each individual case.

\section{PATIENT'S CONSENT:}

Informed consent was obtained from the patient.

\section{CONFLICT OF INTEREST:}

Authors declared no conflict of interest.

\section{AUTHORS' CONTRIBUTION:}

CGK: Authored surgical procedure.

ISG: Carried out orthodontic treatment of the patient and penned that part of the manuscript.

\section{REFERENCES}

1. Isik BK, Esen A, Menziletoglu D. Cerrahi destekli hizli maksiller genisletme: Bir derleme. Atatürk Üniversitesi Dis Heimligi. Fakültesi. Dergisi 2016; 16:141-6.

2. Holberg C, Steinhauser S, Rudzki I. Surgically assisted rapid maxillary expansion: Midfacial and cranial stress distribution. Am J Orthod Dentofacial Orthop 2007; 132:776-82.

3. Hernandez-Alfaro F, Mareque Bueno J, Diaz A, Pages CM. Minimally invasive surgically assisted rapid palatal expansion with limited approach under sedation: A report of 283 consecutive cases. J Oral Maxillofac Surg 2010; 68:2154-8.

4. Glassman AS, Nahigian SJ, Medway JM, Aronowitz HI. Conservative surgical orthodontic adult rapid palatal expansion: Sixteen cases. Am J Orthod 1984; 86:207-13.

5. Northway WM, Meade JB. Surgically assisted rapid maxillary expansion: A comparison technique, response, and stability. Angle Orthodontics 1997; 67:309-20.

6. Berger JL, Pangrazio-Kulbersh V, Borgula T, Kaczynski R. Stability of orthopedic and surgically assisted rapid palatal expansion over time. Am J Orthod Dentofacial Orthop 1998; 114:638-45.

7. Pereira MD, Prado Ribeiro GP, Abramoff Fracher MM, Aloise AC, Ferreira LM. Classification of midpalatal suture opening after surgically-assisted rapid maxillary expansion using computed tomography. Oral Surg Oral Med Oral Pathol Oral Radiol Endod 2010; 110:41-5.

8. Pogrel MA, Kaban LB, Vargervik K, Baumrind S. Surgicallyassisted rapid maxillary expansion in adults. Int J Adult Orthodon Orthognath Surg 1992; 7:37-41.

9. Zandi M, Miresmaeli A, Heidari A, Lamei A. The necessity of pterygomaxillary disjunction in surgically-assisted rapid maxillary expansion: A short-term, double blind, historical controlled clinical trial. J Craniomaxillofac Surg 2016; 44:1181-6.

10. Seeberger R, Kater W, Davids R, Thiele OC. Long term effects surgically assisted rapid maxillary expansion without performing osteotomy of the pterygoid plates. J Craniomaxillofac Surg 2010; 38:175-8. 